\title{
Total Body Photography and Sequential Digital Dermoscopy in Pregnant Women
}

\author{
Gabriela M. Martins-Costa ${ }^{1}$, Renato Bakos ${ }^{1,2}$
}

1 Postgraduate Program in Medical Sciences, Universidade Federal do Rio Grande do Sul (UFRGS), Brazil

2 Department of Dermatology, Hospital de Clínicas de Porto Alegre (HCPA), Universidade Federal do Rio Grande do Sul (UFRGS), Brazil

Key words: dermoscopy, melanocytic nevi, pregnancy, digital monitoring, total body photography

Citation: Martins-Costa GM, Bakos R. Total body photography and sequential digital dermoscopy in pregnant women. Dermatol Pract Concept. 2019;9(2):126-131. DOI: https://doi.org/10.5826/dpc.0902a08

Accepted: January 30, 2019; Published: April 30, 2019

Copyright: @2019 Martins-Costa and Bakos. This is an open-access article distributed under the terms of the Creative Commons Attribution License, which permits unrestricted use, distribution, and reproduction in any medium, provided the original author and source are credited.

Funding: None.

Competing interests: The authors have no conflicts of interest to disclose.

Authorship: Both authors have contributed significantly to this publication.

Corresponding author: Gabriela M. Martins-Costa, MSc, Rua Goncalo de Carvalho, 221/301, Floresta, Porto Alegre, RS, Brazil, 900350170. Email: gmynarskimc@gmail.com

ABSTRACT Background: Melanocytic nevi can vary in size and number in pregnant women, and the differential diagnosis with melanoma may be challenging.

Objectives: To describe changes in total body photography of pregnant women and dermoscopy aspects of their nevi.

Methods: A prospective cohort study with 703 melanocytic nevi from 18 women was performed, comparing them in the first and third trimester of pregnancy. Images were analyzed between the 2 periods for changes in dermoscopic aspects.

Results: Total body photography images indicated that $44 \%$ of patients had new lesions. Regarding the observed changes, there were symmetric or regular changes of the network ( $23 \%$ of cases), occurrence of new globules/dots $(12.4 \%)$, new vascular structures $(3.2 \%)$, new streaks $(1.7 \%)$, and new structureless area $(1.0 \%)$. Moreover, $55.0 \%$ of the nevi increased in size. Enlarging of the nevi was observed mostly on the abdomen $(87.1 \% ; \mathrm{P}<0.001)$ and showed more network changes $(27.1 \% ; \mathrm{P}=$ $0.014)$ and formation of new globules and dots $(16.0 \% ; \mathrm{P}<0.001)$. Patients with a risk of developing melanoma presented more frequently enlarged nevi $(45 \% ; \mathrm{P}=0.019)$. The association between streak formation and skin type was significant $(\mathrm{P}=0.012)$ and was more frequent in skin type II $(2.7 \%)$, when compared with skin types III $(1.3 \%)$ and IV $(0 \%)$.

Conclusions: Development of new melanocytic nevi may occur in pregnant women. The majority of the preexisting melanocytic nevi showed enlarging, and most of them presented with benign dermoscopic changes. The appearance of new streaks is more frequent in fair skin types. Patients with a personal or family history of melanoma in first-degree relatives presented more nevi with changes in size. 


\section{Introduction}

Pregnancy is known as one of the clinical scenarios capable of inducing dynamic dermoscopic changes in melanocytic nevi $[1,2]$. Some other factors are age, skin type, and ultraviolet radiation exposure. A few studies on dermoscopic alterations in melanocytic nevi during pregnancy have already showed an increase in size, darkening and/or lightening of lesions, variations of pigmented network, appearance of dots or globules, and increased vascularization as possible changing features $[2,3]$.

Recently, melanoma in pregnant women has been reported to be associated with increased mortality when compared with nonpregnant women [4-6]. Therefore, it is crucial to recognize physiological or benign dermoscopic changes in melanocytic nevi and differentiate them from potential malignant transformation.

Most studies that have been published on dermoscopic changes of nevi during the gestational period have evaluated only a few lesions from each patient [2,7,8-15]. As far as we know there are no reports of total body photography in pregnant women. The aim of the present study was to evaluate the occurrence of new melanocytic lesions in pregnant women and to describe dermoscopic changes in preexisting nevi using total body photography and digital dermoscopy follow-up.

\section{Methods}

Pregnant women with a minimum of 10 nevi were consecutively recruited to the study. The patients were invited to participate after a regular visit at our department or a regular prenatal visit at the Department of Gynecology and Obstetrics. Informed consent was obtained at the beginning of the observational period according to an institutional review board-approved protocol. The visits were performed in the first and third trimesters of gestation. Total body photography and digital dermoscopy from the majority of the patient's melanocytic nevi were taken, using a nonpolarized light video dermatoscope (Fotofinder; Fotofinder Systems, Tübingen, Germany). Digital images between the two evaluations were then compared by one of the authors; equivocal lesions were also analyzed by a second observer. Melanocytic nevi were defined as pigmented lesions $2 \mathrm{~mm}$ or more in size and presenting a nevus-compatible dermoscopic pattern.

A total of 703 melanocytic nevi were evaluated and analyzed for changes in size and for changes in dermoscopic features (network, streaks, globules and dots, new structureless area, pigment pattern, and vascularization). Changes in size and shape were evaluated by the automated FotoFinder Moleanalyzer system and were given in square millimeters. We considered pigment network changes associated with the pregnancy-nevus behavior: expansion (increase in size) with or without darkening and thickening in a symmetric distribution. The other dermoscopic structure changes were also considered to be physiological when they occurred also in a symmetric or regular distribution. Lesions that presented network and other dermoscopic structures changing in an asymmetric or irregular distribution were considered for excision.

In addition, we tested the association of total body photography and dermoscopic changes with patients' clinical features, such as skin type, presence of relevant melanoma risk factors (high-risk patients for melanoma were those with personal or family history of melanoma in first-degree relatives), and age.

Descriptive statistics were obtained for continuous data using mean and standard deviation with chi-square tests. For the categorical data, the numbers and percentages were analyzed with t test and Wald chi-square tests. At the data crossing, we used the contingency tables with the chi-square test adjusted with generalized estimating equations. The study significance level was set at $\alpha=0.05(\mathrm{P}<0.05)$. Data were analyzed with SPSS version 22.0.

\section{Results}

We evaluated total body photography of 18 pregnant women and digital dermoscopy of 703 melanocytic nevi. We observed a mean of 43.5 nevi per patient (ranging from 9 to 69). Patients' ages ranged from 28 to 44 years. The mean age was 33 years $(\mathrm{SD}=3.49)$.

The mean number of pregnancies was $1.44(\mathrm{SD}=0.70)$. Four patients $(4 / 18 ; 22.2 \%)$ had a positive family history of melanoma and one patient had a personal history of melanoma. Regarding skin types, 9/18 patients (50\%) had skin type III, $7 / 18$ patients ( $33.3 \%$ ) had type II, and $3 / 18$ patients $(16.6 \%)$ had type IV. Thirteen patients had some degree of freckling and solar lentigines $(13 / 18 ; 72.2 \%)$. The most frequent global dermoscopic pattern was the reticular global pattern, present in approximately 16/18 (88.9\%) patients.

When comparing total body photography images between the first and third trimester, 8/18 (44\%) patients presented new melanocytic nevi, ranging from 1 to 5 new lesions per patient. Several body sites were involved, but all patients with new lesions presented at least one of them on the upper limbs. Patients younger than 30 years of age presented more frequently new melanocytic nevi formation when compared with patients older than $30(6 / 16 ; 37.5 \%$; $\mathrm{P}<0.001)$ (Table 1$)$.

Regarding sequential dermoscopic evaluation of nevi, $55 \%(387 / 703)$ of the nevi enlarged when the first and third trimesters were compared. Seventy-three of 703 (10.4\%) had increased pigmentation. Considering baseline dermoscopic structures in preexisting nevi, pigmented network was the structure most frequently showing changes (163/703; 
$23.2 \%)$. We observed new vascular structures in 23 lesions; they were dotted or comma-like vessels, regularly distributed. New streaks were visualized in only 12 lesions, also symmetrically distributed in the lesion or at the periphery. Data on new melanocytic lesions and modifications in preexisting nevi are shown in Table 2.

When analyzing an association between enlargement of nevi with clinical aspects and presence of dermoscopic structures, we observed that the majority of the enlarged nevi were located at the abdomen $(88 / 101 ; 87.1 \%$; $<0.001)$ in comparison with other body locations. We could not find an association between enlargement of nevi and skin types ( $\mathrm{P}=$ 0.959) and age $(P=0.867)$. Patients with a risk of developing melanoma presented with more nevi that increased in size (99/220; 45\%; P = 0.019). Enlarged nevi presented typical network changes $(105 / 387 ; 27 \% ; \mathrm{P}=0.014)$ and globule/dot formation $(62 / 387 ; 16 \%$; $<$ 0.001) (Table 3).

Regarding the association of dermoscopic changes in preexisting nevi and association with skin types, patients with the fair skin type had more streak formation than did those with dark skin types III and IV $(\mathrm{P}=0.012)$. There was no correlation between different skin types and other dermoscopic changes. There was no association between high-risk patients for melanoma and occurrence of new dermoscopic structures or changes, such as network change $(P=0.915)$, globule/dot formation $(\mathrm{P}=0.816)$, or streaks $(\mathrm{P}=0.320)$ (data not shown).

\section{Discussion}

The use of digital follow-up and dermoscopy to evaluate pigmented lesion changes in pregnancy is certainly the best approach in early detection of melanoma in pregnancy in high-risk patients for melanoma. They are noninvasive tools and do not carry any fetal risk [13,16].

Pigmentary changes, such as melasma and enlargement of nevi, are frequently reported by pregnant women [17]. In our study we observed an occurrence of new melanocytic nevi in $44 \%$ of the patients. In all pregnant women with new lesions, at least one of them occurred on the upper limbs (Figure 1). Despite pregnancy effects, we suggest that sun exposure may play a major role in this association. Previous reports described single nevus modifications during pregnancy $[8,13,16,17]$. Duarte et al reported a new lesion in the nipple of a pregnant woman that involuted to its baseline size 2 months after delivery [8]. Zampetti et al described modifications of a preexisting nevus on the nose [13]. Although we had only 2 patients younger than 30 years, we could observe that pregnant women at this age presented more new lesions than older women. To our knowledge, this is the first study to report the occurrence of new benign melanocytic lesions evaluated by total body photography in a series of pregnant
Table 1. Clinical Characteristics of New Lesions During Total Body Photography

\begin{tabular}{|l|c|}
\hline \multicolumn{1}{|c|}{ Clinical Characteristics } & n (\%) \\
\hline Patients with new lesions & $8(44.4)$ \\
\hline No. of new lesions per patient \\
\hline 1 lesion & $2(11.0)$ \\
\hline 2 lesions & $2(11.0)$ \\
\hline$\geq 3$ lesions & $4(17.0)$ \\
\hline Location of new lesions $(\mathrm{n}=21)$ & $8(100.0)$ \\
\hline Upper limbs & $3(37.5)$ \\
\hline Chest & $3(37.5)$ \\
\hline Abdomen & $3(37.5)$ \\
\hline Lower limbs & $2(25.0)$ \\
\hline Back & $1(12.5)$ \\
\hline Head & $1(12.5)$ \\
\hline Neck &
\end{tabular}

Table 2. Frequencies of Dermoscopic Changes Between First and Third Trimester Observed in 703 Melanocytic Nevi

\begin{tabular}{|l|c|}
\hline \multicolumn{1}{|c|}{ Dermoscopic Aspects } & n (\%) \\
\hline Enlargement & $387(55.6)$ \\
\hline Pigmentation \\
\hline Hyperpigmentation & $73(10.4)$ \\
\hline Hypopigmentation & $41(5.8)$ \\
\hline Dermoscopic structures \\
\hline Network changes & $163(23.2)$ \\
\hline New dots/globules & $87(12.4)$ \\
\hline New vascular structures & $23(3.2)$ \\
\hline New streaks & $12(1.7)$ \\
\hline New structureless areas & $7(1.0)$ \\
\hline
\end{tabular}

women. Meanwhile, few reports have described pigmentary changes in lesions that were further diagnosed as melanomas $[9,15]$. The authors emphasized that during follow-up these lesions presented asymmetric changes in shape and size as well as occurrence of new dermoscopic structures such as starburst pattern, streaks, black dots, dark red areas, and blue-gray pigmentation that increased the suspicion of a malignant behavior.

We demonstrated that nevi may go through changes during pregnancy. The changes occur generally in a symmetric and regular fashion. We observed in our study that $55 \%$ of preexisting nevi enlarged in the third trimester, mainly on the abdomen in comparison with other body sites $(\mathrm{P}<0.001)$ (Figure 2). In line with our data, Strumia et al evaluated nevi of 12 pregnant women and found a major enlargement 
Table 3. Association of Nevus Enlargement With Body Location, Skin Type, Melanoma Risk, Presence of Dermoscopic Structures, and Age Group ( $\mathbf{n}=703)$

\begin{tabular}{|l|r|r|}
\hline \multicolumn{3}{|c|}{ n (\%) } \\
\hline Body location & $88 / 101(87.1)$ & $<0.001$ \\
\hline Abdomen & $100 / 178(56.2)$ & \\
\hline Back & $79 / 146(54.1)$ & \\
\hline Anterior chest & $58 / 114(50.9)$ & \\
\hline Lower limbs & $19 / 39(48.7)$ & \\
\hline Neck & $10 / 28(35.7)$ & \\
\hline Face & $29 / 93(31.2)$ & \\
\hline Upper limbs & $32 / 54(59.3)$ & 0.959 \\
\hline Skin type & $142 / 255(55.7)$ & 0.019 \\
\hline IV & $213 / 394(54.1)$ & 0.014 \\
\hline II & $99 / 220(45.0)$ & $<0.001$ \\
\hline III & & 0.765 \\
\hline High-risk patients for melanoma ${ }^{a}$ & $105 / 387(27.1)$ & \\
\hline Dermoscopic structures & $62 / 387(16.0)$ & \\
\hline Network changes & $6 / 387(1.6)$ & \\
\hline Globules/dots formation & & \\
\hline Streaks & $9 / 16(56.3)$ & \\
\hline Age group (n = 18) & & \\
\hline$<30$ yrs old & & \\
\hline$>30$ yrs old & & \\
\hline
\end{tabular}

aPatients presenting with personal or family history of melanoma in first-degree relatives.
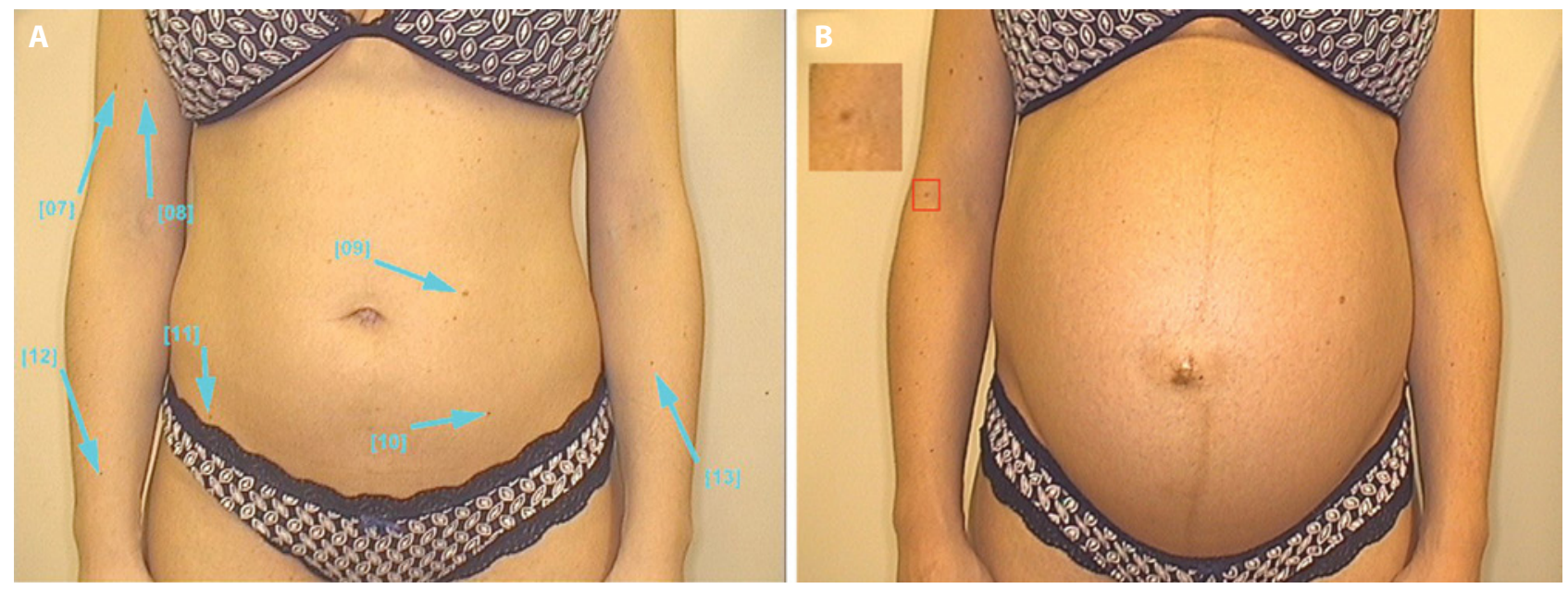

Figure 1. (A) Baseline image of the anterior portion of the trunk and arms of a patient during the first trimester. (B) The development of a new lesion in the right arm in the third trimester (red square) is highlighted in the left upper corner. Blue arrows are not relevant for this evaluation. They are markers in the system that we used which are necessary to number nevi that will be evaluated by dermoscopy. [Copyright: (C)2019 Martins-Costa and Bakos]

of nevi in pregnancy-related expanding areas, showing that body site is the most significant factor for nevus enlargement in pregnancy [7]. Moreover, Zampino et al studied only nevi on the back of 47 pregnant women and they did not observe alteration in size [10]. Aktürk et al noticed a statistically significant difference between the mean diameters of nevi in the first and third trimester $(\mathrm{P}=0.001)$. The enlargements were more significant on the anterior portion of the body, although they did not reach a statistically significant difference. They observed, however, a statistically significant increase in nevus Total Dermoscopy Score (TDS) mean when comparing the first and third trimesters $(P=0.008)$ [9-11]. 


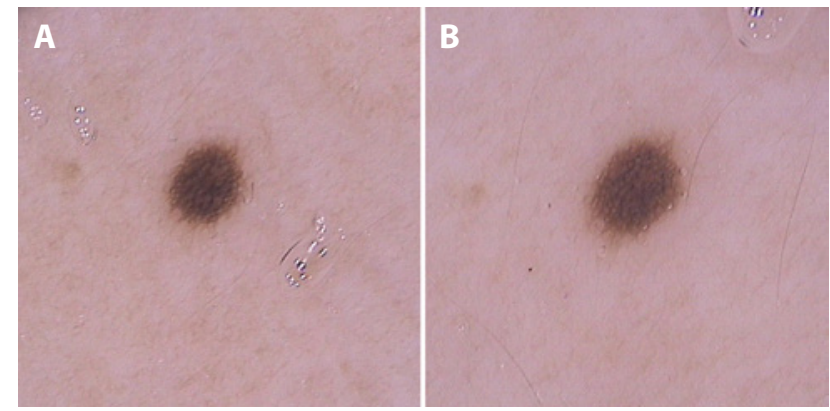

Figure 2. (A) Dermoscopic image (20 x original magnification) of a melanocytic lesion in the first trimester on the lateral trunk. (B) Image from the same nevus in the third trimester showing enlargement with a symmetric and homogeneous network distribution. [Copyright: (C2019 Martins-Costa and Bakos]

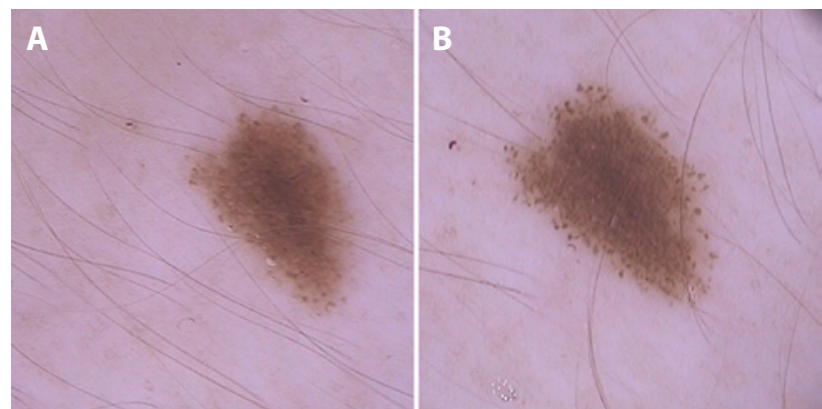

Figure 3. (A) Dermoscopic image $(20 \times$ original magnification) of a melanocytic lesion in the first trimester on the middle of the torso. (B) Image from the same nevus in the third trimester showing a rim of symmetric and homogeneous distributed dots in the periphery of the lesion. [Copyright: (C2019 Martins-Costa and Bakos]
High-risk patients for melanoma also presented more changing nevi in comparison with low-risk patients. Several studies have reported that those patients show more "unstable" nevi. This finding reinforces that pregnant women with multiple nevi and past or family history of melanoma should be closely monitored during pregnancy [17-20]. Moreover, the occurrence of new or changing melanocytic lesions in pregnancy showing an irregular or asymmetric pattern should be prompted for excision to rule out melanoma [6,13,20].

Pigmented network symmetric thickening/enlargement and new development of globules/dots in a regular distribution were the most common dermoscopic alterations found in the study (Figure 3). Homogeneous network enlargement and well-distributed globules and dots may be associated with physiological changes in melanocytic nevi of young people. These are dermoscopic features generally associated with growth in melanocytic nevi [20-24]. Regarding these dermoscopic changes, Rubegni et al observed a more prominent pigment network with architectural disorder and darkening of globules [14]. In the postpartum period the globules faded, but the lesions showed persisting changes in network. Moreover, Gunduz et al studied the nevi of 21 pregnant women and reported a TDS increase of $19 \%$ in the lesions. Major dermoscopic changes were darkening and increase of globules/dots (in number and in size), thickening of the pigmented network, and radial streaming formation [12]. Zampino et al observed a progressive lightening of the nevi at the end of pregnancy and after delivery. The lesions showed persisting changes in network. At the end of pregnancy, vessels increased and a higher TDS was observed, with a significant reduction in both after delivery [10]. Although not frequent, we observed streaks and structureless area formation in our study [12-16]. It is important to state that new structures occurred in a symmetric and regular distribution.

Patients with fair skin usually have more nevi than those with dark skin types. We observed that new streaks were more frequent among pregnant women with fair skin, although we did not observe any other association regarding dermoscopic changes of preexisting nevi and skin types [19].

During the follow-up period we decided to remove only 1 lesion. It was a new, fast-growing, atypical melanocytic lesion in the third trimester in a patient with a family history of melanoma and a history of progesterone use during the first weeks of gestation (Figure 4). The histopathological analysis reported dysplastic melanocytic nevi (Figure 4). Irregular hyperpigmentation areas have recently been described as one of most relevant dermoscopic structures in differentiating atypical nevi from in

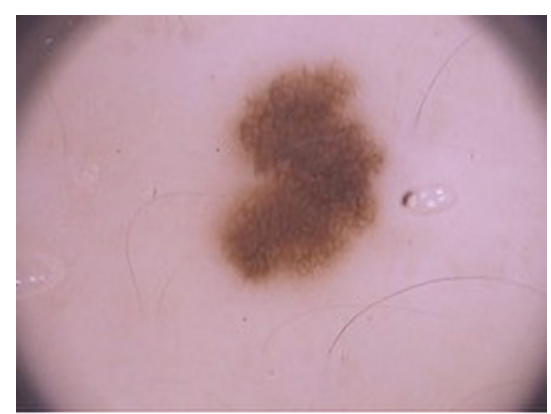

Figure 4. Dermoscopic image showing a new irregular pigmented lesion in the third trimester on the left buttock $(20 \times$ original magnification). Histopathological examination revealed an atypical nevus. [Copyright: (02019 Martins-Costa and Bakos]

situ melanoma. Therefore, we reinforce the need for removing lesions with this kind of dermoscopic picture during pregnancy [23].

\section{Conclusions}

The limitation of this study is the low number of patients included, although we believe that the results presented can be representative of melanocytic nevi during pregnancy. Moreover, pregnancy occurs usually in young women. It is known that melanocytic nevi may vary in size and number at this age. Although we did not include a control group of nonpregnant women paired by age, we also believe that our data reflect dynamic changes in melanocytic nevi associated with pregnancy and not 
only with age. These observations are probably transitory due to physiological changes and might not affect lifetime nevus count [24].

Finally, pregnancy is clearly the major clinical scenario in which melanocytic nevi might present clinical and dermoscopic changes. In this paper we point out some modifications that pregnant women may present in total body photography and sequential dermoscopy. We demonstrate that a significant portion of pregnant women develop new lesions. In addition, symmetric and regular enlargement in the size, network changes, and new formation of globules/dots were the major alterations observed in preexisting nevi. Further studies comparing total body photography and dermoscopic images of melanocytic nevi in pregnant women and nonpregnant control women might help in elucidating the impact of other factors for dynamic changes in nevi during pregnancy.

\section{Acknowledgments}

The authors thank Dr. Inês Alencar de Castro (In Memoriam) for her valuable contribution to this work.

\section{References}

1. Zalaudek I, Docimo G, Argenziano G. Using dermoscopic criteria and patient-related factors for the management of pigmented melanocytic nevi. Arch Dermatol. 2009;145(7):816-826.

2. Borges V, Puig S, Malvehy J. Melanocytic nevi, melanoma, and pregnancy [in Spanish]. Actas Dermosifiliogr. 2011;102(9):650657.

3. Sanchez JL, Figueroa LD, Rodriguez E. Behavior of melanocytic nevi during pregnancy. Am J Dermatopathol. 1984;6 Suppl:89-91.

4. Stensheim H, Moller B, van Dijk T, Fossa SD. Cause-specific survival for women diagnosed with cancer during pregnancy or lactation: a registry-based cohort study. J Clin Oncol. 2009;27(1):4551.

5. Moller H, Purushotham A, Linklater KM, et al. Recent childbirth is an adverse prognostic factor in breast cancer and melanoma, but not in Hodgkin lymphoma. Eur J Cancer. 2013;49(17):36863693.

6. Byrom L, Olsen C, Knight L, Khosrotehrani K, Green AC. Increased mortality for pregnancy-associated melanoma: systematic review and meta-analysis. J Eur Acad Dermatol Venereol. 2015;29(8):1457-1466.

7. Strumia R. Digital epiluminescence microscopy in nevi during pregnancy. Dermatology. 2002; 205(2):186-187.

8. Duarte AF, Correia O. Pregnancy breast lesions: the role of dermoscopy [abstract P1703]. J Am Acad Dermatol. 2009; 60(suppl): AB89.
9. Sato T, Ishiko A, Saito M, Tanaka M, Ishimoto H, Amagai M. Rapid growth of malignant melanoma in pregnancy. J Dtsch Dermatol Ges. 2008;6(2):126-129.

10. Zampino MR, Corazza M, Costantino D, Mollica G, Virgili A. Are melanocytic nevi influenced by pregnancy? A dermoscopic evaluation. Dermatol Surg. 2006;32(12):1497-1504.

11. Aktürk AS, Bilen N, Bayramgurler D, et al. Dermoscopy is a suitable method for the observation of the pregnancy-related changes in melanocytic nevi. J Eur Acad Dermatol Venereol. 2007;21(8):1086-1090.

12. Gunduz K, Koltan S, Sahin MT, E Filiz E. Analysis of melanocytic naevi by dermoscopy during pregnancy. J Eur Acad Dermatol Venereol. 2003;17(3):349-351.

13. Zampetti A, Feliciani C, Landi F, et al. Management and dermoscopy of fast-growing nevi in pregnancy: case report and literature review. J Cutan Med Surg. 2006;10(5):249-252.

14. Rubegni P, Sbano P, Burroni M, et al. Melanocytic skin lesions and pregnancy: digital dermoscopy analysis. Skin Res Technol. 2007;13(2):143-147.

15. Canosa JM, Brechtbühl ER, Duprat Neto JP, Rezze GG. Dermatoscopy in pregnancy [in Portuguese]. Surg Cosmet Dermatol [Internet]. 2011;3(3):261-263. Available at: http://www.redalyc. org/articulo.oa?id=265522087016. Accessed May 24, 2018.

16. Campos-do-Carmo G, Ramos-e-Silva M. Dermoscopy: basic concepts. Int J Dermatol. 2008;47(7):712-719.

17. Kar S, Krishnan A, Shivkumar PV. Pregnancy and skin. J Obstet Gynaecol India. 2012;62(3):268-275.

18. Haenssle HAMN, Ngassa A, Buhl T, et al. Association of patient risk factors and frequency of nevus-associated cutaneous melanomas. JAMA Dermatol. 2016;152(3):291-298.

19. Tuma B, Yamada S, Atallah AN, Araujo FM, Hirata SH. Dermoscopy of black skin: a cross-sectional study of clinical and dermoscopic features of melanocytic lesions in individuals with type V/VI skin compared to those with type I/II skin. J Am Acad Dermatol. 2015;73(1):114-119.

20. Fikrle T, Pizinger K, Szakos H, et al. Digital dermatoscopic followup of 1027 melanocytic lesions in 121 patients at risk of malignant melanoma. J Eur Acad Dermatol Venereol. 2013;27(2):180186.

21. Enninga EA, Holtan SG, Creedon DJ, et al. Immunomodulatory effects of sex hormones: requirements for pregnancy and relevance in melanoma. Mayo Clin Proc. 2014;89(4):520-535.

22. Holtan SG, Creedon DJ, Haluska P, Markovic SN. Cancer and pregnancy: parallels in growth, invasion, and immune modulation and implications for cancer therapeutic agents. Mayo Clin Proc. 2009;84(11):985-1000.

23. Lallas A, Longo C, Manfredini M, et al. Accuracy of dermoscopic criteria for the diagnosis of melanoma in situ. JAMA Dermatol. 2018;154(4):414-419.

24. MacKie RM, English J, Aitchison TC, Fitzsimons CP, Wilson P. The number and distribution of benign pigmented moles (melanocytic naevi) in a healthy British population. Br J Dermatol. 1985;113(2):167-174. 